\title{
Ubiquitous Sensorization for Multimodal Assessment of Driving Patterns
}

\author{
Fábio Silva, Cesar Analide, Celestino Gonçalves and João Sarmento \\ University of Minho \\ Department of Informatics \\ \{fabiosilva, analide\}@di.uminho.pt, celestin@ipg.pt, joao.sarmento@gmail.com
}

\begin{abstract}
Sustainability issues and sustainable behaviours are becoming concerns of increasing significance in our society. In the case of transportation systems, it would be important to know the impact of a given driving behaviour over sustainability factors. This paper describes a system that integrates ubiquitous mobile sensors available on devices such as smartphones, intelligent wristbands and smartwatches, in order to determine and classify driving patterns and to assess driving efficiency and driver's moods. It first identifies the main attributes for contextual information, with relevance to driving analysis. Next, it describes how to obtain that information from ubiquitous mobile sensors, usually carried by drivers. Finally, it addresses the multimodal assessment process which produces the analysis of driving patterns and the classification of driving moods, promoting the identification of either regular or aggressive driving patterns, and the classification of mood types between aggressive and relaxed. Such an approach enables ubiquitous sensing of personal driving patterns across different vehicles, which can be used in sustainability frameworks, driving alerts and recommendation systems.
\end{abstract}

Keywords: Driving Profile, Mobile Sensors, Sustainability

\section{Introduction}

Ambient Intelligence (AmI) is a very active area of knowledge and constitutes a multi-disciplinary subject which takes advantage of advances in sensing systems, pervasive devices, context recognition, and communications. Nowadays, AmI applications can be found in fields ranging from home, office, transport, tourism, recommender and safety systems, among many others [20]. In the case of transport applications, an area also known as Smart Cars [2], the AmI system must be aware not only of the car situation, but also of the driver's intention, of his physical and physiological conditions and of the best way to deal with them [19], [17]. The driver's behaviour is, thus, of key importance: several authors have used machine learning and dynamical graphical models for modelling and recognizing driver's behaviours [22].

There are examples of applications integrating AmI and ubiquitous principles in driving and traffic analysis. In [13], it is described a monitoring and analysis 
system to approach personalized driving behaviour, for emerging hybrid vehicles. The system is fully automated, non-intrusive with multi-modality sensing, based on smartphones. The application runs while driving and it will present personalized quantitative information of the driver's specific driving behaviour. In [18] a mobile application assesses driving behaviour, based on critical driving events, giving feedback to the driver. The Nericell system [15], from Microsoft Research, monitors road and traffic conditions using the driver's smartphone and corresponding incorporated sensors, but it can also detect honking levels and potholes on roads. The I-VAITS project [19] is an example that pretends to assist the driver appropriately and unobtrusively, analysing real-time data from the environment, from the car and from the driver itself, by the way the driver uses the different elements of the car, their movements or image processing of their face expressions. In [3], in the context of a car safety support system, an ambient agent-based model for a car driver behaviour assessment is presented. The system uses sensors to periodically obtain information about the driver's steering operation and the focus of the driver's gaze. In the case of abnormal steering operation and unfocused gaze, the system launches proceedings in order to slow down, stop the car and lock the ignition.

\section{Related Work}

Driving analysis can be a complex problem depending on the degree of information used and the number of categories being analysed. The analysis presented is described based on sustainable principles assessing driving patterns and their impact on sustainability and sustainable behaviour.

\subsection{Driving Pattern Detection}

Usually, driving pattern is defined and associated to the speed profile of the driver, but it can be expanded to other variables, as gear changing, and big changes on the acceleration [6]. In 1978, Kuhler and Karstens [12] introduced a set of ten driving pattern parameters. Later, in 1996, André [1] reviewed those parameters, and reviewed some of the most common parameters such as action duration, speed, acceleration, idle periods and number of stops per kilometre. In other studies [5], [6] other parameters were used to collect data from ordinary drivers in real traffic situations, such as wheel rotation, engine speed, ambient temperature, use of breaks and fuel-use. In these studies, GPS data was also monitored, where each driving pattern was attributed to street type, street function, street width, traffic flow and codes for location in the city (central, semi-central, peripheral). It was concluded that the street type had the most influence on the driving pattern. The analysis of the 62 primary calculated parameters, resulted in 16 independent driving pattern factors, each describing a certain dimension of the driving pattern. When investigating the effect of the independent driving pattern factors on exhaust emissions, and on fuel consumption, it was found that only 9 factors had a significant effect. 
Table 1: Relevant attributes to driving analysis according to previous studies

\begin{tabular}{|c|c|c|c|}
\hline Attribute & Ericsson [5] & Kuhler and Karstens [12] & Nericell [15] \\
\hline Wheel rotation & + & + & - \\
Motor RPM & + & + & - \\
Pedals Monitoring & + & + & - \\
Street type & + & + & + \\
Fuel Consumption & + & + & + \\
Velocity & + & + & + \\
Acceleration & + & + & + \\
Standard deviation of acceleration & + & + & + \\
Trip duration & + & + & + \\
Hour of day & + & + & \\
\hline
\end{tabular}

Table 1 provides an analysis of the main attributes identified. These studies share most of the identified attributes to analyse and classify driving patterns. With exception of attributes such as motor rotations per minute and pedals monitoring that are obtained directly from the physical vehicles, driving analysis with mobile and non-mobile sensors take interest in the same subgroup of attributes.

\subsection{Sustainable Driving}

Computational methods that allow the balancing of economic, environmental and social factors needed to a sustainable development, a newly emerging and interdisciplinary area, known as Computational Sustainability, solve problems which are essentially decision and optimization problems. The concept of sustainability and sustainable behaviours is important to ensure the welfare and well-being. Due to its importance, some researchers have discussed about quantification methods, and modelling sustainability [23], [11]. In the case of transportation systems, the assessment of the impact of a given driving pattern is made over sustainability factors, like fuel consumption, greenhouse gas emissions, dangerous behaviour or driving stress.

A system to estimate a driver profile using smartphone sensors, able to detect risky driving patterns, is proposed in [4]. It was verified whether the driver behaviour is safe or unsafe, using Bayesian classification. It is claimed that the system will lead to fuel efficient and better driving habits. In [9], and in addition to car sensory data, physiological data was continuously collected and analysed (heart rate, skin conductance, and respiration) to evaluate a driver's relative stress. The CarMa, Car Mobile Assistant, is a smartphone-based system that provides high-level abstractions for sensing and tuning car parameters, whereby developers can easily write smartphone applications. The personalized tuning can result in over $10 \%$ gains in fuel efficiency [7]. The MIROAD system, Mobile-Sensor-Platform for Intelligent Recognition Of Aggressive Driving [10], is a mobile system capable to detecting and recognizing driving events and driving patterns, intending to increase awareness and to promote safety driving, and thus 
possibly achieving a reduction in the social and economic costs of car crashes. The system uses Dynamic Time Warping and smartphone based sensor-fusion to detect and recognize actions without external processing.

\section{Multimodal Assessment System}

The implementation of the ubiquitous multimodal driving analysis system is depicted in this section. Ubiquitous monitoring is achieved by the use of smartphones equipped with accelerometer, GPS, compass, microphone and light sensors. These come as standard in most smartphones sold today. While it is not the main function of a smartphone, driving analysis can be achieved using some of the limited processing capability of low-end smartphones. Additionally, its connectivity options allow for better analysis on a server side location. The proof-of-concept system is illustrated by figure 1, where data flow is illustrated.

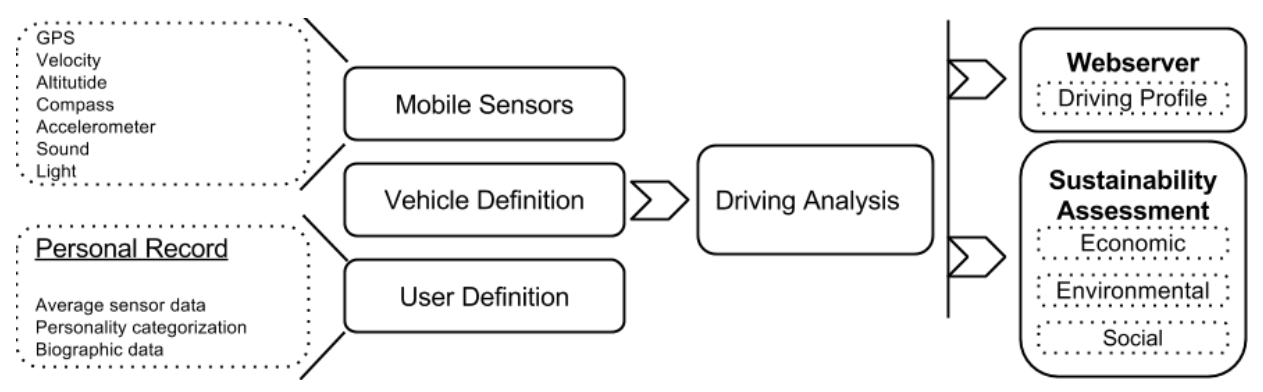

Fig. 1: Model describing the driving analysis in the system.

After obtaining information about driving patterns, vehicle and driver definition, the driving analysis module will synchronize its data with an external server and derive a report assessment on the sustainable impact according to its 3 dimensions is built. Such knowledge is useful to update sustainability frameworks such as PHESS [21] which monitor and assess sustainable impact through performance indicators based on the same three categories.

The economic and environmental assessment is derived from the estimated impact of current driving patterns on the vehicle fuel consumption and gas emission. The social component is assessed by the effect of driving patterns on the social and psychological response from drivers. Although, sensor analysis by itself can answer if an event can or cannot be considered aggressive, it is still a reactive and instantaneous concept. With such information it is possible to map optimal to suboptimal configurations as well as infer which emotions these conditions produce on human beings. In a computational system it is still a challenging process to acquire rich information in this domain, but there are some approaches proposed in the research community that have had broad acceptance. In regard to the representation of a personality, the OCEAN structure used is similar to 
the approach adopted by the ALMA framework to represent the personality of people and initiate mood states in [8]. In the OCEAN approach, personality are defined by a set of variables $(o, c, e, a, n)$ which represent five personality traits: Openness, Conscientiousness, Extraversion, Agreeableness and Neuroticism. On the other hand, the PAD space [14], is a computational friendly representation of mood states. A person's mood is represented using the three variables that define PAD space, Pleasure, Arousal and Dominance respectively. Contrary to the personality which is almost regarded as static during people lifetimes, mood is a temporal state of the human mind that can last for minutes, hours or even days.

\subsection{User Driving Pattern}

The analysis of driving patterns is made with the help of profiles. These structures are created individually for each driver and maintained in a web-server through the use of restful web-services. Although not using the internal data from vehicle sensor as in past research [5], the approach followed in this work uses smartphone data for ubiquitous and pervasive monitoring. An illustration of the application devised to record driver's attributes is detailed in the left part of figure 2. Data gathering is made through sensors, which is pre-processed internally with data fusion methodologies to enrich data and provide richer information. The number of variables used to assess driving patterns is based on the information gathered in the literature and adapted to ubiquitous sensors. As such, a total of 6 basic attributes are monitored: accelerometer, velocity, altitude, time of day, compass and position. From the fusion of these attributes, it is possible to infer standard deviations for each attribute according to each driver, the number of breaking and accelerations and its mean duration and intensity. These are the characteristics used in each driver profile in order to assess its regular driving behaviour. Aside from the regular driving pattern, the system will also classify aggressive driving patterns, which are categorized by higher frequency of breaks and accelerations with high intensity and shorter duration than the driver regular behaviour. The rigth part of figure 2 shows a graphical
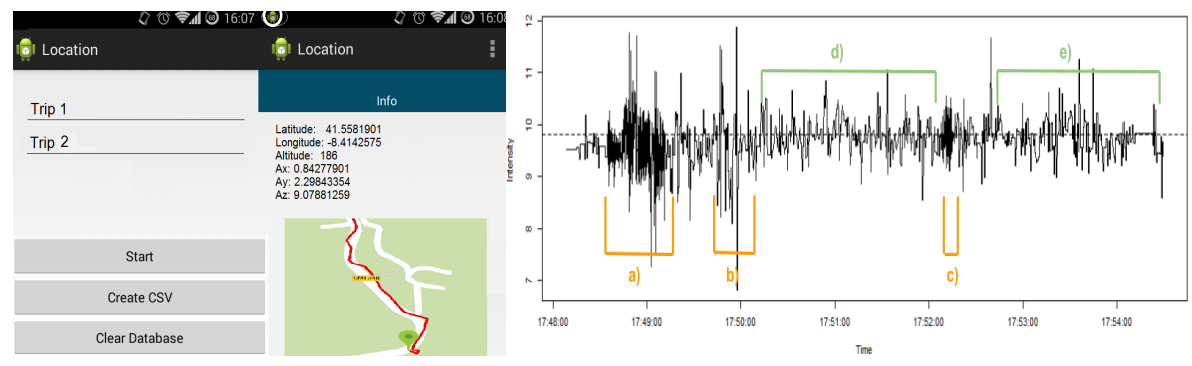

Fig. 2: Mobile Driving Patterns Extraction 
representation of the intensity of the accelerometer sensor annotated with information gathered by other sensors according to driving pattern of a test driver. Event $a$ ) represents low velocity and high variation of accelerometer which can be deduced to be parking or congested traffic driving. Events b), c) represent aggressive events where velocity was kept high and sudden changes of direction result in high amplitude variation of accelerometer forces. Events $d$ ), e) do not offer significant variation from common driving pattern thus identified as regular driving events. It is the identification of these events that will play a major role to detect the current mood of the driver. The pattern identified and its duration will provide information to assess the current driver's mood.

\subsection{Driving Mood Classification}

Mood classification is based on the analysis of the number of breaks and accelerations detected by mobile sensors: aggressive driving styles are connected with high frequency of breaking and accelerating actions; relaxed driving is correlated with stable velocity and low breaking and accelerating actions. The computational representation of mood states is done according to the Pleasure, Arousal, Dominance framework described by Mehabian (PAD) [14] and the PAD space extended by Gehbard in [8], where the initial $P, A, D$ variables are initiated according to each user's personality assessment. The initial assessment of a driver's personality is achieved by an initial questionnaire, filled the first time the mobile sensing application is used. In this case the Newcastle Personality Assessor (NPA) questionnaire was used [16].

$$
M_{\text {actual }}=M_{\text {actual }}+\left(M_{\text {final }}-M_{\text {actual }}\right) * \text { emotionalWeight }
$$

Following this approach, two final states are defined representing aggressive and calm states so that a driver's mood may be updated towards one of these states. The equation 1 represents the current driver's state moving in a vector space at a velocity defined by the emotionalWeigth. This is dependent on values from the OCEAN personality representation and the assessment of the emotional response to a driving pattern. If positive update (relaxed pattern) emotionalWeight $=(e+1.0) *$ const otherwise emotionalWeight $=$ $(n+1.0) *$ const.

This study is limited to a classification of mood states oscillating between aggressive and relaxed moods. The classification of additional mood types increases not only complexity but also the error rate, as it becomes difficult to distinguish between them.

\section{Conclusions and Future Work}

In this article it is present an analysis of some state of the art related ubiquitous sensing of driving patterns. The approach with this work allows for pervasive monitoring through common objects such as smartphones in order to record and 
analyse driver's actions. Although limited by the sensors of each device, realist results can be extracted by standard hardware. The assessment of current driving mood is robust to sporadic deviant events as only a series of continuous negative or positive patterns does change the mood assessment while still reacting instantly to aggressive or relaxed events.

As a future work, the ubiquitous application will be extended allowing the sharing of information between drivers. With these abilities, gamification elements will try to moderate driving patterns by adding positive and negative points to a driver's profile. Additionally, the information shared allows for instantaneous information about nearby drivers such as emotion classification. The analysis will also add route classification based on an aggregated knowledge of driving patterns for each route, as well a It will also be used in future recommender systems and navigation applications. It will also allow automatic identification of driving actions through sensor analysis, thus requiring no user input at the start of driving records, enhancing the ubiquitous and pervasive nature of this work.

\section{acknowledgements}

This work is part-funded by ERDF - European Regional Development Fund through the COMPETE Programme (operational programme for competitiveness) and by National Funds through the FCT - Fundação para a Ciência e a Tecnologia (Portuguese Foundation for Science and Technology) within project FCOMP-01-0124-FEDER-028980 (PTDC/EEI-SII/1386/2012). It is also supported by a doctoral grant, SFRH/BD/78713/2011, issued by FCT in Portugal.

\section{References}

1. André, M.: Driving Cycles Development: Characterization of the Methods. Tech. rep., INRETS (May 1996)

2. Aztiria, A., Izaguirre, A., Augusto, J.C.: Learning patterns in ambient intelligence environments: a survey. Artif. Intell. Rev. 34(1), 35-51 (May 2010)

3. Bosse, T., Hoogendoorn, M., Klein, M.C.A., Treur, J.: A Component-Based Ambient Agent Model for Assessment of Driving Behaviour. In: Sandnes, F., Zhang, Y., Rong, C., Yang, L., Ma, J. (eds.) Ubiquitous Intelligence and Computing, Lecture Notes in Computer Science, vol. 5061, pp. 229-243. Springer Berlin Heidelberg (2008)

4. Eren, H., Makinist, S., Akin, E., Yilmaz, A.: Estimating driving behavior by a smartphone. In: 2012 IEEE Intelligent Vehicles Symposium. pp. 234-239. No. 254, IEEE (Jun 2012)

5. Ericsson, E.: Variability in exhaust emission and fuel consumption in urban driving. Urban Transport Systems. Proceedings from the 2nd kfb Research Conference (1980), 1-16 (2000)

6. Ericsson, E.: Independent driving pattern factors and their influence on fuel-use and exhaust emission factors. Transportation Research Part D: Transport and Environment 6(5), 325-345 (2001) 
7. Flach, T., Mishra, N., Pedrosa, L., Riesz, C., Govindan, R.: CarMA. In: Proceedings of the 9th ACM Conference on Embedded Networked Sensor Systems - SenSys '11. p. 135. ACM Press, New York, New York, USA (2011)

8. Gebhard, P.: ALMA: a layered model of affect. Proceedings of the fourth international joint conference on Autonomous agents and multiagent systems pp. 29-36 (2005)

9. Healey, J., Picard, R.: Detecting Stress During Real-World Driving Tasks Using Physiological Sensors. IEEE Transactions on Intelligent Transportation Systems 6(2), 156-166 (Jun 2005)

10. Johnson, D.A., Trivedi, M.M.: Driving style recognition using a smartphone as a sensor platform. In: 2011 14th International IEEE Conference on Intelligent Transportation Systems (ITSC). pp. 1609-1615. IEEE (Oct 2011)

11. Kharrazi, A., Kraines, S., Hoang, L., Yarime, M.: Advancing quantification methods of sustainability: A critical examination emergy, exergy, ecological footprint, and ecological information-based approaches. Ecological Indicators 37, Part A(0), 81-89 (2014)

12. Kuhler, M., Karstens, D.: Improved Driving Cycle for Testing Automotive Exhaust Emissions. Tech. rep., Volkswagenwerk AG (Feb 1978)

13. Li, K., Lu, M., Lu, F., Lv, Q., Shang, L., Maksimovic, D.: Personalized Driving Behavior Monitoring and Analysis for Emerging Hybrid Vehicles. In: Proceedings of the 10th International Conference on Pervasive Computing. pp. 1-19. Pervasive'12, Springer-Verlag, Berlin, Heidelberg (2012)

14. Mehrabian, A.: Pleasure-arousal-dominance: A general framework for describing and measuring individual differences in temperament. Current Psychology 14(4), 261-292 (1996)

15. Mohan, P., Padmanabhan, V.N., Ramjee, R.: Nericell. In: Proceedings of the 6th ACM conference on Embedded network sensor systems - SenSys '08. p. 323. ACM Press, New York, New York, USA (2008)

16. Nettle, D.: Personality: What makes you the way you are. OUP Oxford (2007)

17. Oliveira, T., Novais, P., Jose, N.: Guideline Formalization and Knowledge Representation for Clinical Decision Support. Advances in Distributed Computing and Artificial Intelligence Journal (ADCAIJ) I(2), 1-12 (2012)

18. Paefgen, J., Kehr, F., Zhai, Y., Michahelles, F.: Driving Behavior Analysis with Smartphones: Insights from a Controlled Field Study. In: Proceedings of the 11th International Conference on Mobile and Ubiquitous Multimedia. pp. 36:1-36:8. ACM, USA (2012)

19. Rakotonirainy, A., Tay, R.: In-vehicle ambient intelligent transport systems (I-VAITS): towards an integrated research. In: Proceedings. The 7th International IEEE Conference on Intelligent Transportation Systems (IEEE Cat. No.04TH8749). pp. 648-651. IEEE (2004)

20. Sadri, F.: Ambient intelligence. ACM Computing Surveys 43(4), 1-66 (2011)

21. Silva, F., Analide, C., Rosa, L., Felgueiras, G., Pimenta, C.: Ambient Sensorization for the Furtherance of Sustainability. In: Ambient Intelligence-Software and Applications, pp. 179-186. Springer (2013)

22. Sun, J., Wu, Z.h., Pan, G.: Context-aware smart car: from model to prototype. Journal of Zhejiang University SCIENCE A 10(7), 1049-1059 (Jul 2009)

23. Todorov, V., Marinova, D.: Modelling sustainability. Mathematics and Computers in Simulation 81(7), 1397-1408 (2011) 